\title{
Efficacy of a new dietary supplement in dogs with advanced chronic kidney disease
}

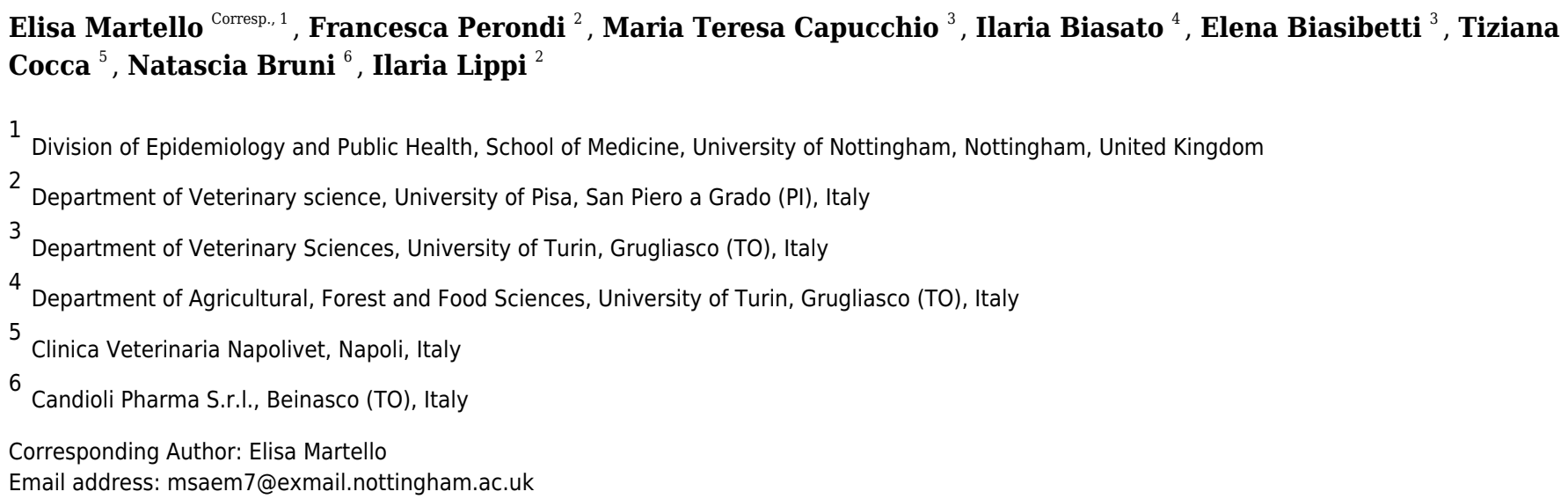

Chronic kidney disease (CKD) is a common disease in elderly dogs. The present study aims to evaluate the efficacy of a dietary supplement containing calcium carbonate, calciumlactate gluconate, chitosan and sodium bicarbonate in dogs with IRIS stage 3 of CKD. Twenty dogs were enrolled in the study, ten were administered the new dietary supplementation for 180 days ( $T$ group) while the others were used as control group (C group). Haematologic, biochemical and urinalysis were performed every 30 days. A significant reduction in the $T$ group compared to the $C$ group in serum phosphorus level and increase in serum bicarbonate and ionized calcium values were recorded. The urine protein-to-creatinine ratio (UPC) was significantly lower in the T group at the end of the study compared to the $\mathrm{C}$ group. The tested supplement could be considered as a supportive treatment for dogs with advanced CKD. 
2

3 Elisa Martello ${ }^{1 *}$, Francesca Perondi², Maria Teresa Capucchio ${ }^{3}$, Ilaria Biasato ${ }^{4}$, Elena Biasibetti ${ }^{3}$, Tiziana

4 Cocca $^{5}$, Natascia Bruni ${ }^{6}$, Ilaria Lippi ${ }^{2}$

5

$6 \quad{ }^{1}$ Division of Epidemiology and Public Health, School of Medicine, University of Nottingham, UK

72 Department of Veterinary science, University of Pisa, San Piero a Grado (PI), Italy

$8{ }^{3}$ Department of Veterinary Sciences, University of Turin, Grugliasco (TO), Italy

$9 \quad{ }^{4}$ Department of Agricultural, Forest and Food Sciences, University of Turin, Grugliasco (TO), Italy

$10{ }^{5}$ Clinica Veterinaria Napolivet, Napoli, Italy

$11{ }^{6}$ Candioli Pharma S.r.I., Beinasco (TO), Italy

12

$13 *$ Corresponding Author:

14 Dr. Elisa Martello ${ }^{1}$

15 Division of Epidemiology and Public Health, School of Medicine, University of Nottingham

16 Email address: msaem7@exmail.nottingham.ac.uk

17 Phone: +447510999441

18 ORCID ID: 0000-0003-0247-6670

19 


\section{Abstract}

21 Chronic kidney disease (CKD) is a common disease in elderly dogs. The present study aims to evaluate the

22 efficacy of a dietary supplement containing calcium carbonate, calcium-lactate gluconate, chitosan and

23 sodium bicarbonate in dogs with IRIS stage 3 of CKD. Twenty dogs were enrolled in the study, ten were

24 administered the new dietary supplementation for 180 days (T group) while the others were used as

25 control group (C group). Haematologic, biochemical and urinalysis were performed every 30 days. A

26 significant reduction in the $T$ group compared to the $C$ group in serum phosphorus level and increase in

27 serum bicarbonate and ionized calcium values were recorded. The urine protein-to-creatinine ratio (UPC)

28 was significantly lower in the $T$ group at the end of the study compared to the $C$ group. The tested

29 supplement could be considered as a supportive treatment for dogs with advanced CKD.

Keywords.

32 Chronic kidney disease, dog, dietary supplement, serum phosphorus, serum bicarbonate 


\section{Introduction.}

Chronic kidney disease (CKD) is generally defined as any structural and/or functional abnormality, which can affect one or both kidneys and has been present in patients for at least three months. Chronic kidney disease is a very common disease in canine population, with a high prevalence especially in older patients. Generally, the progression of the disease is slow, with a survival time from months to a year or two (Bartges 2012; Davies 2016; Polzin 2011; Smets et al. 2010). The management of CKD is focused on slowing down the progression of the disease by controlling the major risk factors and clinical signs. In veterinary medicine there are several parameters to be monitored overtime in patients with CKD in order to modify the therapy when needed. The major factors to monitor are: proteinuria, hypertension and hyperphosphatemia.

Renal diets specifically designed for CKD aims to slow down progression of the disease and extend survival, to improve clinical consequences of uremia and minimize electrolyte and acid-base unbalance, and maintain adequate nutritional intake (Biasibetti et al. 2018; Jacob et al. 2002; Polzin 2013). The formulation of canine renal diets has changed and improved over the years, the administration of this food is considered the first therapeutic approach for improving survival and life quality of canine patients with CKD, using a four-stage scale of disease progression according to the International Renal Interest Society (IRIS) guidelines ((IRIS) 2019; Davies 2016). Nutritional support is also essential to control the protein and phosphate intake to avoid the risk of malnutrition and dehydration and to reduce mortality (Rudinsky et al. 2018). When the diet alone is not sufficient to control phosphate and/or metabolic acidosis or to slow down the progression of CKD, the use of dietary supplements (i.e. phosphate binders and alkalizing agents) is a useful strategy adopted by veterinarians (Lippi et al. 2017; Polzin 2011; Zatelli et al. 2017).

The clinicians have to choose the most appropriate therapeutic approach to maintain serum phosphorus concentration within the range reported by the IRIS, according to each CKD stage ). Currently, there is a 
58 wide choice of commercially available phosphate binders which, through mechanisms of chelation, are

59 able to prevent the absorption of phosphorus by the intestinal mucosa, through the formation of inert

60 compounds directly eliminated with the faeces. Metabolic acidosis is another promoter for the progression of CKD and for impairing protein nutrition (de Brito-Ashurst et al. 2009; Polzin 2013). With CKD, there is increased retention of metabolic acids, increased production of ammonia, and decreased production of bicarbonate. Metabolic acidosis occurs in less than $10 \%$ of cats with stage 2 or 3 CKD and in 50\% with uremia (Bartges 2012; Polzin 2013), but to our knowledge no data is available for prevalence in dogs with CKD. Metabolic acidosis is associated with hyporexia/anorexia, hypokalemia, and muscle weakness (Bartges 2012). In human medicine, therapy with bicarbonate has been reported to slow down the progression of CKD and to improve the nutritional and health status Raphael 2019;. Metabolic alkalinizing agents are commonly used to increase the blood $\mathrm{pH}$ given the ability to bind $\mathrm{H}+$ ions. Alkalization therapy is indicated for dogs with IRIS CKD Stages 1-4 when blood pH and bicarbonate concentration drop below the normal range. When diet alone is insufficient, administration of alkalinizing agents such as potassium citrate and sodium bicarbonate is indicated (IRIS) 2019; Polzin 2013; Zatelli et al. 2017; Zatelli et al. 2012).

In dogs with CKD, commercial dietary supplements with different compounds such as chitosan calcium carbonate, and potassium citrate show their beneficial effect to control hyperphosphatemia (Zatelli et al. 2012). A recent study on cats testing an oral supplement with calcium carbonate, calcium-lactate gluconate, chitosan and also sodium bicarbonate confirms the reduced serum phosphorus and the increase in serum bicarbonate with improvement of clinical conditions (Biasibetti et al. 2018).

The present study aims to evaluate the ability to bind phosphate and correct metabolic acidosis, together with the ease of administration of a new commercial dietary supplement containing calcium carbonate, calcium-lactate gluconate, chitosan and sodium bicarbonate in dogs with CKD. 


\section{Materials \& Methods.}

82 A prospective, randomised, controlled study on dogs affected by CKD was performed in 2015. The

83 Veterinary clinic Napolivet (Naples, Italy) database was screened in order to include dogs with advanced

CKD stages 3 and/or 4 according to the International Renal Interest Society (IRIS). Diagnosis of CKD was

performed by persistently azotemia by previous laboratory findings and/or estimated from the medical

history (>3 months of polyuria/polydipsia), physical examination findings consistent with chronic disease

(loss of weight and lean body mass, small kidney size), or evidence of chronic structural abnormalities identified by ultrasound imaging (small kidneys, renal infarcts, renal fibrosis). Dogs included were classified following guidelines developed by IRIS, based on serum creatinine concentration, the magnitude of proteinuria as measured by the urine protein, creatinine ratio (UPC) and blood pressure (BP) (IRIS, 2013).

Dogs suspected or affected by other concomitant diseases (acute kidney injury, pre-renal or post-renal azotemia, genitourinary tract inflammation or infection, urinary tract obstruction, heart disease, chronic heart failure, neoplasia, hypothyroidism, diabetes) assessed by clinical, instrumental and laboratory evaluations, were excluded from the study.

The dogs' owners were informed about the purpose and the design of the study and signed a written informed consent. All procedures, treatments and animal care were in compliance with the guidelines of the Italian Minister of Health for the care and use of animals (D.L. 4 March 2014 n. 26 and D.L. 27 January 1992 n.116) and UE (Directive 86/609/CEE) and the use of supplements was regulated by the Regulation (EC) No 767/2009.

After the enrollment in the study (TO), dogs were randomly assigned to two groups: the control group (C 102 group, $n=10$ ) and the treated group (T group, $n=10)$. History, physical examination including body weight 103 (BW) and body condition score (BCS) (1 to 5 scoring system) were recorded. Complete blood count (CBC), 
105

106

107

108

109

110

111

112

113

114

115

116

117

118

119

120

121

122

124

125

126

127

128

an electronic sphygmomanometers (CONTEC), during the examination, measurements were taken five times and the mean value was recorded. Urinalysis procedures were already described in Biasibetti and colleagues (Biasibetti et al. 2018). A complete clinical examination, blood and urinary tests were performed at the beginning of the study (T0), then at day 30 (T30), 60 (T60), 90 (T90), 120 (T120), 150 (T150) and 180 (T180). All the animals were fed with the same commercial renal diet (Royal Canin Renal Canine) for the whole duration of the trial and from at least eight weeks before the inclusion in the study.

The amount offered to each dog was based on the estimated metabolic requirements (calorie intake) according to the FEDIAF Nutritional Guidelines (Fédération européenne de l'industrie des aliments pour animaux familiers (FEDIAF), 2014). In the T group a new supplement produced by the Candioli Pharma S.r.I. ("Renal P") containing calcium carbonate, calcium-lactate gluconate, chitosan and sodium bicarbonate (Table 1) was added to the commercial renal diet. The daily supplement dosage was set as $0.2 \mathrm{~g} / \mathrm{kg}$ body weight, and it was divided in two daily administrations mixed with the two meals, so as to favor the reduction of intestinal phosphorus absorption. The supplement was given for a total of 180 days. nata.

Dogs with clinical signs (vomiting, poor appetite) and/or proteinuria, hypertension, were started on appropriate treatment weeks to months prior to T0. Owners were asked to monitor daily eposodes of vomiting, diarrhea, anorexia during the entire duration of the study.

Dogs with persistent proteinuria were treated with benazepril (Fortekor; Novartis Animal Health, Varese, Italy), 0.25 to $0.5 \mathrm{mg} / \mathrm{kg}$ body weight (PO) once to twice daily. Dogs with a history of hypertension (BP > $160 \mathrm{mmHg}$ ) and/or with the risk of extra-renal target organ damage (TOD) were maintained on a combination of benazepril and amlodipine (Amodip; CEVA Salute Animale), 0.25 to $0.5 \mathrm{mg} / \mathrm{kg} \mathrm{BW}$ (PO) once daily as recommended by IRIS staging system (IRIS 2013).

Blood examination

During each examination, blood examination and urinalysis were performed.

Peer) reviewing PDF | (2019:12:44209:1:1:NEW 26 Jun 2020) 
129 The laboratory performed CBC (Animal blood counter, Scil -Vet abcTM). Serum biochemical analysis

130 (Automatic Analyzer-Echo, Edif) recorded the values of: blood urea nitrogen (BUN), creatinine (CREA),

131 phosphorus (P), total protein (TP), albumin (ALB), albumin/globulin ratio (A/G), glucose (GLU), alanine

132 aminotransferase (ALT), aspartate aminotransferase (AST), alkaline phosphatase (ALP), bilirubin (BIL), and

133 cholesterol (CHOL). Venous blood gas analysis was also performed in all cases with an analytical device

134 (Abaxis VetScan i-Stat1) in order to assess bicarbonate (HCO3) and ionized calcium (iCA).

135 Urinalysis.

136 Urine samples were obtained by cystocentesis with a $5 \mathrm{~mL}$ syringe. They were put in a sterile collection

137 tube and analyzed. Urine sediment was obtained by centrifugation ( $2 \mathrm{~min}$ at $1500 \mathrm{xg}$ ) followed by removal

138 of supernatant which was stored at $+4{ }^{\circ} \mathrm{C}$. For the urine sediment examination and specific gravity test

139 an in-house refractometer was used. To calculate the UPC, protein and creatinine concentrations $(\mathrm{mg} / \mathrm{dL})$

140 were measured with the pyrogallol red method and Jaffe method, respectively, within 12 hours from the

141 collection. Dipstick analysis was used for the urine test strip.

142 Dogs were classified as non-proteinuric, borderline proteinuric, or proteinuric according to the IRIS

143 substaging system (UPC $<0.2=$ non-proteinuric, UPC 0.2 to $0.5=$ borderline proteinuric, and UPC $>0.5=$

144 proteinuric).

145 Statistical analysis

146 GraphPad Prism ${ }^{\circledR}$ software was used to perform statistical analysis. Shapiro-Wilk test established the 147 normality or non-normality distribution of data. Repeated measures ANOVA (post-hoc test: Bonferroni's 148 Multiple Comparison Test) and Friedman (post-hoc test: Dunn's Multiple Comparison test) tests were 149 used to perform the intra-group comparisons among the experimental times, while the inter-group 150 comparisons between groups were performed by Student $t$ and Mann-Whitney $U$ tests (with $p$-values 151 corrected to account for multiple comparisons). Data are expressed as mean and SEM. Significance was 152 set as $p<0.05$ (intra-group comparisons) or $p<0.008$ (inter-group comparisons). 
Results.

155 A total of 20 dogs with CKD IRIS stage 3 were recruited and all dogs ( $n=10$ T group; $n=10$ C group) completed the study. The median age of all dogs was 10 years. Dogs' ages ranged from 5 to 15 years (mean $10.7 \pm 2.9 \mathrm{SD}$ ) in the T group and from 8 to 14 years (mean $10.1 \pm 1.8 \mathrm{SD}$ ) in the $\mathrm{C}$ group. In the T group, four dogs were intact males and six were females ( $50 \%$ sterilized), while in the $C$ group six dogs were intact males and four were females ( $50 \%$ sterilized). Eight dogs were mixed breed, two were German Shepherd, then one was Maltese, one Belgian Shepherd, one Yorkshire terrier, one Levriero, one Fox Terrier, one Jack Russel Terrier, one Schnauzer, one Beagle, one Dobermann and one Basset hound. At the time of inclusion as well as at the end of the study, there were no statistical differences between $(p>0.008)$ or ( $p>0.05$ ) within groups with regards to BW and BCS (Table 2).

Hematological values (Table 3) were recorded during the entire study. All the hematological parameters were also similar between the two groups for all the considered experimental times ( $>0.008)$. Furthermore, no significant differences were recorded for blood pressure between or within groups at the beginning and at the end of the study $(p>0.05)$ (Table 4).

All the biochemical parameters measured during the study are reported in Table 5. Most of the parameters remained stable during the trial in both groups. However, an increase in blood urea nitrogen $(B U N)(p<0.05)$ and creatinine $(C R E A)(p<0.05)$ was reported in the $C$ group, while BUN remained stable and CREA progressively decreased in the T group during the study period $(p<0.05)$. Even so, all these parameters were similar between the two groups for all the considered experimental times $(p>0.008)$. No difference in the serum phosphorus level between the two groups was reported at day 0 ( $p>0.008)$.

174 This value significantly decreased at each time point from day 30 to $180(p<0.008)$ in the T group compared 175 to the $C$ group (Figure 1 ). In addition, in the $C$ group all dogs, at each time point, showed levels of serum 176 phosphorus above the normal range $(2.7-5 \mathrm{mg} / \mathrm{dl})$, while in seven dogs in the T group the same level came 
177 back to normal within 30 days after the beginning of the treatment, in the other three at T60 $(n=1)$ and

178 T90 $(n=2)$ and it remained so during the course of the treatment. Considering the mean values, a 179 significant progressive reduction overtime was reported in the T group $(p<0.05)$ (Figure 1, Table 5).

180 No difference in the serum iCa level between the two groups was observed at day $0(p>0.008)$. A significant

181 increase of this parameter was observed from day $120(p<0.008)$ in the T group compared with the C 182 group, but it remained within the normal range $(1.29-1.41 \mathrm{mmol} / \mathrm{l})$, while the mean iCa level in the $\mathrm{C}$ group 183 remained stable during the study and below the reference range (Table 6, Figure 2).

184 No difference in the serum $\mathrm{HCO}_{3}$ level between the two groups was reported at day $0(p>0.008)$. Serum $185 \mathrm{HCO}_{3}$ was lower than the normal range $(18-24 \mathrm{mmol} / \mathrm{l})$ in both groups at T0 (Table 6$)$, but limited to the T 186 group we observed a significant increase in values during the trial from day 30 on $(p<0.005)$. As a result, 187 at day $180, \mathrm{HCO}_{3}$ was significantly greater $(p<0.008)$ in the T group than in the $\mathrm{C}$ group (Table $6 \mathrm{~A}$, Figure 188 3), but mean values were still below the reference range.

189 At the baseline, all dogs $(n=10)$ of the $C$ group and eight dogs in the T group were proteinuric (UPC $>0.5)$, two were borderline proteinuric (UPC 0.2-0.5) (Table 7). However, no differences in the UPC between the two groups were observed at day 0 ( $p>0.008)$. On the contrary, urine protein, creatinine ratio at days 150 and 180 was lower $(p<0.008)$ in the T group compared to the C group (Figure 4). No differences in UPC, were found within the same group during the treatment period $(p<0.05)$.

All the dogs remained in IRIS stage 3 for the duration of the study. All the owners in the T group, reported the ease of administration of the supplement which was entirely consumed as specifically indicated in the study protocol.

All the selected animals completed the study and all the owners reported a good palatability of the tested feed supplementation product. No adverse effects (vomiting, diarrhea, anorexia) monitored daily by the owner were observed during the whole study period. 
201

202

203

204

205

206

207

208

209

210

211

212

213

214

215

216

217

218

219

220

221

222

223

\section{Discussion.}

The main aim of the present study was to determine the efficacy of a new dietary supplement administered for a period of 180 days in dogs in IRIS stage 3 . In the present study, the time of observation was longer (180 vs 28 days) than another study where a similar supplement contained chitosan, enteric phosphate binders, and alkalinizing agents was used (Zatelli et al. 2012), it is a great advantage in terms of monitoring the long-term impact and safety of the product. The supplement administration helped reducing the mortality rate due to uremic crises in dogs with CKD, but it failed to show significantly improvement for mean serum concentrations of creatinine, BUN, phosphate and $\mathrm{HCO}_{3}$ (Zatelli et al. 2012). In addition, the values were recorded at 4-8 weeks following the supplement administration (Zatelli et al. 2012). A more recent study, showed that another type of dietary supplement reduced significantly serum phosphorus and increased $\mathrm{HCO}_{3}$ values in cats with $\mathrm{CKD}$, improving their clinical conditions, without any adverse reaction (Biasibetti et al. 2018). In our study, the dogs treated with dietary supplementation showed a significant reduction in serum phosphorus level and an increase in $\mathrm{HCO}_{3}$ compared to the control group (Figure 1). In particular, mean serum phosphorus levels started to show a significant reduction by day 30 since the administration of the tested supplementation in T group: these dogs showed a progressive reduction of serum phosphorus at different time intervals $(p<0.01)$ within the group and compared to the $\mathrm{C}$ group. In the present study the tested supplement has proved to be effective, considering that the serum phosphorus in the T group came back to normal $(2.7-5 \mathrm{mg} / \mathrm{dL})$ in all dogs within 90 days (12 weeks) or even before, from the beginning of the treatment (Figure 1).

In our study, the $90 \%$ of patients at the time of inclusion in the trial, had a blood bicarbonate concentration below $18 \mathrm{mmol} / \mathrm{l}$ and at the end of the study $(T=180)$, the concentration increased significantly $(p<0.01)$ in the $T$ group compared with the $C$ group (Figure 1, Table 2), but it did not normalize. Although the supplement was able to increase serum concentration of bicarbonate at the recommended dosage, 
224 severe conditions of metabolic acidosis may require higher dosages, or extra supplementation of

225 bicarbonate, in order to achieve the goal of a serum bicarbonate concentration $>18 \mathrm{mmol} / \mathrm{l}$.

226 As CKD dogs usually require both alkalizing and phosphorus binding therapy, the use of a single

227 supplement which includes both agents, like the one we tested, has the advantage to facilitate the

228 owner's compliance. A significant increase of iCa was found in T group at T120, 150 and 180 ( $p<0.01)$

229 compared to the C group (Figure 1). In literature, hypercalcemia has been reported in animals receiving

230 calcium-based phosphate binders, and it is considered as an adverse event (Biasibetti et al. 2018). The

231 phosphate binders used in our supplement were chitosan, calcium lactate gluconate and calcium

232 carbonate. Even if we recorded an increase in iCa, it was not associated with the development of

233 hypercalcemia in the treated dogs. Values remained within the normal range $(1.29-1.41 \mathrm{mmol} / \mathrm{l})$ in both

234 dogs with or without a slight hypocalcaemia at the time of the enrolment, indeed we didn't report any

235 adverse clinical sign linked to that condition. At the beginning of the study, only three dogs in the $\mathrm{C}$ group

236 were also slightly hypercalcaemic and remained so during the trial.

237 In general, ionized hypercalcemia and ionized hypocalcemia was reported in dogs and cats with 238 spontaneous CKD ((Polzin 2011; Schenck \& Chew 2003). Hypercalcemia may occur in dogs with CKD as a 239 consequence of excessive dosages of calcium-containing intestinal phosphate-binding agents (such as

240 calcium acetate, calcium carbonate, or calcium citrate), or in patients with advanced CKD with severe

241 renal hyperparathyroidism ((Finco et al. 1992; Nagode et al. 1996)). Hypercalcemia promotes kidney injury

242 and an increase of the calcium X phosphorus product have been correlated to renal mineralization,

243 inflammation, fibrosis and poor prognosis (Lippi et al. 2014). Lastly, urinalysis showed a significant change

244 in UPC values in the T group when compared to C group, with significantly decrease at the end of the

245 study, specifically at days 150 and $180(p<0.01)$. These findings are different from an analogous study

246 performed on cats testing a similar supplement with calcium carbonate, calcium-lactate gluconate,

247 chitosan and also sodium bicarbonate (Biasibetti et al. 2018), in which UPC showed a significant decrease

Peer] reviewing PDF | (2019:12:44209:1:1:NEW 26 Jun 2020) 
248 already at 30 days from the beginning of the study reflecting an overall improvement of the kidney

249 function in cats treated with alkalinisation therapy. The pathogenetic mechanism is controversial, but it

250 may reflect a possible improvement in glomerular filtration rate (GFR).

251 According to data in human medicine, possible explanations for the reduction of serum concentrations of

252 nitrogen metabolites (BUN and/or CREA), include the compensatory hypertrophy of the functioning

253 nephrons and their enhanced excretion bound of chitosan in the digestive tract. Chitosan can combine

254 acidic substances (i.e. uremic toxins) improving their elimination from the body through faecal material

255 (Zatelli et al. 2012).

256

257 Conclusions

258 In conclusion, our study design has clearly allowed to see the efficacy of the product on a homogenous

259 class of individuals in IRIS stage 3 . The dietary supplementation tested reduced serum phosphorus and

260 increased serum bicarbonate compared to baseline, without causing hypercalcemia, in dogs with CKD IRIS

261 stage 3. It is important to underline that all the animals $(n=10)$ have completed the study and their owners

262 unanimously reported ease of administration of the dietary supplement. Further studies on a larger group

263 of dogs belonging to different IRIS stages would be beneficial.

264

265 Acknowledgements.

266 The study was supported by the Candioli Pharma S.r.l. (Italy). We thank Dr. Mauro Bigliati for reviewing

267 the manuscript.

268 References.

269 (IRIS) International Renal Interest Society. 2013. Guidelines.

270 (IRIS) International Renal Interest Society. 2019. Guidelines. 
271 Bachmann K, Kutter A, Jud Schefer RS, and Sigrist N. 2018. Determination of reference intervals and

272

273

274

275

276

277

278

279

280

281

282

283

284

285

286

287

288

289

290

291

292

293

294 comparison of venous blood gas parameters using a standard and nonstandard collection method in 51 dogs. Schweiz Arch Tierheilkd 160:163-170. 10.17236/sat00150

Bartges JW. 2012. Chronic kidney disease in dogs and cats. Vet Clin North Am Small Anim Pract 42:669692, vi. 10.1016/j.cvsm.2012.04.008

Biasibetti E, Martello E, Bigliati M, Biasato I, Cocca T, Bruni N, and Capucchio MT. 2018. A long term feed supplementation based on phosphate binders in Feline Chronic Kidney Disease. Vet Res Commun 42:161-167. 10.1007/s11259-018-9719-z

Davies M. 2016. Veterinary clinical nutrition: success stories: an overview. Proc Nutr Soc 75:392-397. $10.1017 / \mathrm{s} 002966511600029 x$

de Brito-Ashurst I, Varagunam M, Raftery MJ, and Yaqoob MM. 2009. Bicarbonate supplementation slows progression of CKD and improves nutritional status. J Am Soc Nephrol 20:2075-2084. 10.1681/asn.2008111205

Finco DR, Brown SA, Crowell WA, Duncan RJ, Barsanti JA, and Bennett SE. 1992. Effects of dietary phosphorus and protein in dogs with chronic renal failure. Am J Vet Res 53:2264-2271.

Hutchison AJ, Speake M, and Al-Baaj F. 2004. Reducing high phosphate levels in patients with chronic renal failure undergoing dialysis: a 4-week, dose-finding, open-label study with lanthanum carbonate. Nephrol Dial Transplant 19:1902-1906. 10.1093/ndt/gfh282

Jacob F, Polzin DJ, Osborne CA, Allen TA, Kirk CA, Neaton JD, Lekcharoensuk C, and Swanson LL. 2002. Clinical evaluation of dietary modification for treatment of spontaneous chronic renal failure in dogs. J Am Vet Med Assoc 220:1163-1170. 10.2460/javma.2002.220.1163

Lippi I, Guidi G, Marchetti V, Tognetti R, and Meucci V. 2014. Prognostic role of the product of serum calcium and phosphorus concentrations in dogs with chronic kidney disease: 31 cases (20082010). J Am Vet Med Assoc 245:1135-1140. 10.2460/javma.245.10.1135 
295

297

298

299

300

301

302

303

304

305

306

307

308

309

310

311

312

313

314

315

316

Lippi I, Perondi F, Ceccherini G, Marchetti V, and Guidi G. 2017. Effects of probiotic VSL\#3 on glomerular filtration rate in dogs affected by chronic kidney disease: A pilot study. Can Vet J 58:1301-1305.

Nagode LA, Chew DJ, and Podell M. 1996. Benefits of calcitriol therapy and serum phosphorus control in dogs and cats with chronic renal failure. Both are essential to prevent of suppress toxic hyperparathyroidism. Vet Clin North Am Small Anim Pract 26:1293-1330. 10.1016/s0195$5616(96) 50130-0$

Polzin DJ. 2011. Chronic kidney disease in small animals. Vet Clin North Am Small Anim Pract 41:15-30. 10.1016/j.cvsm.2010.09.004

Polzin DJ. 2013. Evidence-based step-wise approach to managing chronic kidney disease in dogs and cats. J Vet Emerg Crit Care (San Antonio) 23:205-215. 10.1111/vec.12034

Raphael KL. 2019. Metabolic Acidosis in CKD: Core Curriculum 2019. Am J Kidney Dis. 10.1053/j.ajkd.2019.01.036

Rudinsky AJ, Harjes LM, Byron J, Chew DJ, Toribio RE, Langston C, and Parker VJ. 2018. Factors associated with survival in dogs with chronic kidney disease. 32:1977-1982. 10.1111/jvim.15322

Schenck PA, and Chew DJ. 2003. Determination of calcium fractionation in dogs with chronic renal failure. Am J Vet Res 64:1181-1184. 10.2460/ajvr.2003.64.1181

Scherk MA, and Laflamme DP. 2016. Controversies in Veterinary Nephrology: Renal Diets Are Indicated for Cats with International Renal Interest Society Chronic Kidney Disease Stages 2 to 4: The Con View. Vet Clin North Am Small Anim Pract 46:1067-1094. 10.1016/j.cvsm.2016.06.007

Segev G, Bandt C, Francey T, and Cowgill LD. 2008. Aluminum toxicity following administration of aluminum-based phosphate binders in 2 dogs with renal failure. J Vet Intern Med 22:1432-1435. 10.1111/j.1939-1676.2008.0206.x 
317 Smets PM, Meyer E, Maddens BE, Duchateau L, and Daminet S. 2010. Urinary markers in healthy young and aged dogs and dogs with chronic kidney disease. J Vet Intern Med 24:65-72. 10.1111/j.19391676.2009.0426.x

Zatelli A, D'Ippolito P, Roura X, and Zini E. 2017. Short-term effects of dietary supplementation with amino acids in dogs with proteinuric chronic kidney disease. Can Vet J 58:1287-1293.

322

Zatelli A, Pierantozzi M, D'Ippolito P, Bigliati M, and Zini E. 2012. Effect of dietary supplements in reducing probability of death for uremic crises in dogs affected by chronic kidney disease (masked RCCT). 


\section{Table 1 (on next page)}

Composition of the feed supplement used during the study. 
1

\begin{tabular}{|l|l|}
\hline COMPOSITION & g/100g \\
\hline Calcium Lactate Gluconate & 16.00 \\
\hline Calcium carbonate & 26.00 \\
\hline Sodium bicarbonate & 5.00 \\
\hline Chitosan & 8.00 \\
\hline Silica & 0.50 \\
\hline Maltodextrin & 44.30 \\
\hline Sodium pyrophosphate & 0.08 \\
\hline Yeasts [Brewer'yeasts] & 0.05 \\
\hline Lupin meal & 0.02 \\
\hline Salts & 0.02 \\
\hline Sunflower oil & 0.01 \\
\hline TOTAL & $\mathbf{1 0 0}$ \\
\hline
\end{tabular}

2

3 Table 1. Composition of the feed supplement used during the study.

4 


\section{Table 2 (on next page)}

Body weight (BW) and Body Condition Score (BCS) measured during the study in control (C) and treated $(\mathrm{T})$ groups. Data are expressed as mean and standard error of the mean (SEM); T: time in days 


\begin{tabular}{|c|c|c|c|c|c|c|c|}
\hline Parameter & T0 & T30 & T60 & T90 & T120 & T150 & T180 \\
\hline \multicolumn{7}{|c|}{ Group C } \\
\hline BW kg & $21.44 \pm 3.50$ & $21.20 \pm 3.56$ & $20.82 \pm 3.58$ & $20.48 \pm 3.56$ & $23.07 \pm 3.68$ & $22.39 \pm 3.49$ & $21.69 \pm 3.41$ \\
\hline BCS & $2.89 \pm 0.35$ & $2.78 \pm 0.36$ & $2.67 \pm 0.37$ & $2.56 \pm 0.38$ & $2.89 \pm 0.31$ & $2.89 \pm 0.31$ & $2.56 \pm 0.34$ \\
\hline \multicolumn{7}{|c|}{ Group T } \\
\hline BW kg & $17.76 \pm 3.28$ & $17.75 \pm 3.38$ & $17.96 \pm 3.43$ & $17.72 \pm 3.39$ & $17.82 \pm 3.41$ & $17.85 \pm 3.41$ & $17.91 \pm 3.41$ \\
\hline BCS & $2.6 \pm 0.16$ & $2.70 \pm 0.15$ & $2.70 \pm 0.15$ & $2.70 \pm 0.15$ & $2.70 \pm 0.15$ & $2.80 \pm 0.13$ & $2.80 \pm 0.13$ \\
\hline
\end{tabular}

1 Table 2. Body weight (BW) and Body Condition Score (BCS) measured during the study in control 2 (C) and treated (T) groups. Data are expressed as mean and standard error of the mean (SEM); T: 3 time in days

4 


\section{Table 3 (on next page)}

Hematochemical parameters: haematocrit (HCT), haemoglobin (HG), red blood cells (RBC), white blood cells (WBC), neutrophil (N), eosinophil (EO), lymphocytes (LYM) measured during the study in control and treated groups. Data are expressed as mean and stan 


\begin{tabular}{|c|c|c|c|c|c|c|c|c|}
\hline Parameter & $\begin{array}{l}\text { Laboratory } \\
\text { standard } \\
\text { reference } \\
\text { range }\end{array}$ & T0 & $\mathrm{T} 30$ & T60 & T90 & T120 & T150 & T180 \\
\hline \multicolumn{9}{|c|}{ Parameters group C } \\
\hline HCT \% & $37-55$ & $30.76 \pm 2.43$ & $29.86 \pm 2.07$ & $29.71 \pm 2.48$ & $28.40 \pm 2.20$ & $29.87 \pm 2.37$ & $28.42 \pm 2.30$ & $27.59 \pm 2.34$ \\
\hline $\mathrm{RBC} 10^{6} \mathrm{~mm}^{3}$ & $5.5-8.5$ & $5.80 \pm 0.33$ & $5.96 \pm 0.28$ & $5.93 \pm 0.32$ & $5.71 \pm 0.27$ & $5.92 \pm 0.31$ & $5.68 \pm 0.31$ & $5.49 \pm 0.29$ \\
\hline WBC $10^{3} \mathrm{~mm}^{3}$ & $6-17$ & $11.27 \pm 1.09$ & $11.29 \pm 1.12$ & $11.17 \pm 1.63$ & $10.24 \pm 1.25$ & $10.74 \pm 0.94$ & $10.61 \pm 1.25$ & $10.02 \pm 0.68$ \\
\hline $\mathrm{N} 10^{3} \mathrm{~mm}^{3}$ & $3-11.5$ & $7.74 \pm 0.74$ & $7.77 \pm 0.78$ & $7.71 \pm 1.07$ & $6.94 \pm 0.95$ & $7.17 \pm 0.61$ & $7.26 \pm 1.02$ & $6.69 \pm 0.48$ \\
\hline EO $10^{3} \mathrm{~mm}^{3}$ & $0.1-1.3$ & $0.97 \pm 0.07$ & $1.12 \pm 0.12$ & $1.07 \pm 0.17$ & $0.98 \pm 0.10$ & $1.04 \pm 0.16$ & $0.98 \pm 0.08$ & $1.06 \pm 0.08$ \\
\hline HCT \% & $37-55$ & $33.14 \pm 3.61$ & $32.81 \pm 2.82$ & $32.76 \pm 2.62$ & $31.25 \pm 2.71$ & $31.25 \pm 2.57$ & $32.38 \pm 2.56$ & $33.22 \pm 2.50$ \\
\hline $\mathrm{HG} \mathrm{g/dL}$ & $12-18$ & $12.27 \pm 1.24$ & $12.23 \pm 0.93$ & $12.01 \pm 0.85$ & $11.46 \pm 0.83$ & $11.48 \pm 0.78$ & $14.61 \pm 2.72$ & $15.10 \pm 2.77$ \\
\hline $\mathrm{RBC} 10^{6} \mathrm{~mm}^{3}$ & $5.5-8.5$ & $5.72 \pm 0.48$ & $5.67 \pm 0.28$ & $5.65 \pm 0.30$ & $5.49 \pm 0.33$ & $5.51 \pm 0.31$ & $5.80 \pm 0.27$ & $6.04 \pm 0.26$ \\
\hline WBC $10^{3} \mathrm{~mm}^{3}$ & $6-17$ & $11.04 \pm 1.57$ & $10.17 \pm 0.87$ & $8.81 \pm 0.65$ & $8.44 \pm 0.75$ & $9.17 \pm 0.63$ & $9.55 \pm 0.57$ & $9.61 \pm 0.77$ \\
\hline $\mathrm{N} 10^{3} \mathrm{~mm}^{3}$ & $3-11.5$ & $7.83 \pm 1.15$ & $6.88 \pm 0.85$ & $5.81 \pm 0.66$ & $5.86 \pm 0.66$ & $5.83 \pm 0.56$ & $6.54 \pm 0.61$ & $6.11 \pm 0.45$ \\
\hline EO $10^{3} \mathrm{~mm}^{3}$ & $0.1-1.3$ & $0.96 \pm 0.16$ & $1.00 \pm 0.11$ & $0.95 \pm 0.09$ & $1.04 \pm 0.07$ & $1.34 \pm 0.11$ & $1.34 \pm 0.11$ & $1.32 \pm 0.08$ \\
\hline LYM $10^{3} \mathrm{~mm}^{3}$ & $1-4.8$ & $1.09 \pm 0.20$ & $1.35 \pm 0.26$ & $1.28 \pm 0.25$ & $1.23 \pm 0.16$ & $1.39 \pm 0.22$ & $1.35 \pm 0.12$ & $1.23 \pm 0.13$ \\
\hline
\end{tabular}


1 Table 3. Hematochemical parameters: haematocrit (HCT), haemoglobin (HG), red blood cells (RBC), white blood cells (WBC),

2 neutrophil (N), eosinophil (EO), lymphocytes (LYM) measured during the study in control and treated groups. Data are expressed as

3 mean and standard error of the mean (SEM); T: time in days. 


\section{Table 4 (on next page)}

Blood pressure measured during the study. Data are expressed as mean and standard error of the mean (SEM). 
2

\begin{tabular}{|c|c|c|c|c|}
\hline & \multicolumn{2}{|c|}{ T0 } & \multicolumn{2}{c|}{ T180 } \\
\hline & Diastolic (mm Hg) & Systolic (mm Hg) & Diastolic (mm Hg) & Systolic (mm Hg) \\
\hline Group C & $86.67 \pm 3.27$ & $151.67 \pm 4.66$ & $85.00 \pm 1.69$ & $153.75 \pm 3.36$ \\
\hline Group T & $85.00 \pm 2.69$ & $136.00 \pm 5.82$ & $84.00 \pm 1.63$ & $150.50 \pm 2.41$ \\
\hline
\end{tabular}

3 Table 4. Blood pressure measured during the study. Data are expressed as mean and standard error 4 of the mean (SEM). 


\section{Table 5 (on next page)}

Biochemical parameters measured during the study: blood urea nitrogen (BUN), creatinine (CREA), phosphorus (P), total protein (TP), albumin (ALB), albumin/globulin $(A / G)$, glucose $(G L U)$, alanine aminotransferase (ALT), aspartate aminotransferase (AST), alk 


\begin{tabular}{|c|c|c|c|c|c|c|c|c|}
\hline Parameter & Laboratory standard reference range & T0 & $\mathrm{T} 30$ & T60 & T90 & $\mathrm{T} 120$ & $\mathrm{~T} 150$ & $\mathrm{~T} 180$ \\
\hline \multicolumn{9}{|c|}{ Parameters group $\mathrm{C}$} \\
\hline BUN mg/dL & $15-45$ & $120 \pm 10.87^{\mathrm{a}}$ & $132.00 \pm 14.05^{\mathrm{a}}$ & $132.56 \pm 12.54^{\mathrm{a}}$ & $139.00 \pm 10.42^{\mathrm{ab}}$ & $132.00 \pm 9.91^{\mathrm{ab}}$ & $136.00 \pm 9.75^{\mathrm{ab}}$ & $145.44 \pm 13.29^{\mathrm{b}}$ \\
\hline CREA mg/dL & $0.5-1.8$ & $3.14 \pm 0.23$ & $3.16 \pm 0.21$ & $3.17 \pm 0.15$ & $3.33 \pm 0.13$ & $3.29 \pm 0.19$ & $3.36 \pm 0.14$ & $3.46 \pm 0.18$ \\
\hline $\mathrm{P} \mathrm{mg} / \mathrm{dL}$ & $2.7-5$ & $7.81 \pm 0.24$ & $8.09 \pm 0.37$ & $8.14 \pm 0.22$ & $8.32 \pm 0.21$ & $8.00 \pm 0.25$ & $8.16 \pm 0.23$ & $8.23 \pm 0.22$ \\
\hline $\mathrm{TP} \mathrm{mg} / \mathrm{dL}$ & $6-7.5$ & $7.28 \pm 0.43^{\mathrm{a}}$ & $7.94 \pm 0.64^{\mathrm{a}}$ & $7.71 \pm 0.61^{\mathrm{a}}$ & $7.40 \pm 0.58^{\mathrm{ab}}$ & $7.91 \pm 0.68^{\mathrm{ab}}$ & $7.76 \pm 0.60^{\mathrm{ab}}$ & $7.61 \pm 0.61^{\mathrm{b}}$ \\
\hline ALB mg/dL & $2.5-4.2$ & $3.16 \pm 0.13$ & $3.12 \pm 0.12$ & $3.09 \pm 0.08$ & $3.00 \pm 0.07$ & $3.21 \pm 0.09$ & $3.13 \pm 0.08$ & $3.03 \pm 0.06$ \\
\hline $\mathrm{A} / \mathrm{G} \mathrm{mg} / \mathrm{dL}$ & $0.5-1.3$ & $0.91 \pm 0.07$ & $0.82 \pm 0.10$ & $0.83 \pm 0.08$ & $0.84 \pm 0.08$ & $0.79 \pm 0.08$ & $0.84 \pm 0.07$ & $0.82 \pm 0.07$ \\
\hline GLU mg/Dl & $50-100$ & $86.89 \pm 4.00$ & $90.78 \pm 4.45$ & $87.89 \pm 3.15$ & $89.44 \pm 4.49$ & $88.11 \pm 6.32$ & $85.89 \pm 3.55$ & $86.00 \pm 4.63$ \\
\hline ALT UI/L & $7-40$ & $60.89 \pm 10.26$ & $71.89 \pm 19.63$ & $68.33 \pm 16.24$ & $63.89 \pm 15.05$ & $72.00 \pm 12.70$ & $69.67 \pm 11.16$ & $67.11 \pm 10.95$ \\
\hline AST UI/L & $7-40$ & $54.78 \pm 9.21$ & $54.00 \pm 8.38$ & $52.44 \pm 8.53$ & $49.56 \pm 7.07$ & $52.67 \pm 6.94$ & $52.11 \pm 5.79$ & $53.56 \pm 6.44$ \\
\hline ALP UI/L & $5-110$ & $191.78 \pm 22.39$ & $203.89 \pm 26.17$ & $211.33 \pm 30.15$ & $216.33 \pm 32.61$ & $212.67 \pm 25.25$ & $201.67 \pm 23.71$ & $208.33 \pm 20.66$ \\
\hline $\mathrm{BIL} \mathrm{mg} / \mathrm{dL}$ & $0-0.7$ & $0.15 \pm 0.02$ & $0.18 \pm 0.03$ & $0.18 \pm 0.03$ & $0.18 \pm 0.04$ & $0.17 \pm 0.03$ & $0.18 \pm 0.03$ & $0.18 \pm 0.03$ \\
\hline CHOL mg/dL & $140-240$ & $384.67 \pm 23.84$ & $406.33 \pm 24.44$ & $407.89 \pm 23.26$ & $413.78 \pm 25.95$ & $386.56 \pm 24.14$ & $406.22 \pm 30.32$ & $399.33 \pm 27.66$ \\
\hline \multicolumn{9}{|c|}{ Parameters group $\mathrm{T}$} \\
\hline BUN mg/dL & $15-45$ & $124.45 \pm 12.94$ & $110.23 \pm 10.87$ & $107.53 \pm 9.11$ & $115.41 \pm 10.74$ & $123.74 \pm 12.14$ & $119.24 \pm 10.35$ & $124.51 \pm 12.12$ \\
\hline CREA mg/dL & $0.5-1.8$ & $3.52 \pm 0.29^{\mathrm{a}}$ & $3.05 \pm 0.19^{\mathrm{b}}$ & $3.03 \pm 0.16^{\mathrm{b}}$ & $3.15 \pm 0.17^{\mathrm{ab}}$ & $3.14 \pm 0.15^{\mathrm{ab}}$ & $3.12 \pm 0.11^{\mathrm{ab}}$ & $3.19 \pm 0.09^{\mathrm{ab}}$ \\
\hline $\mathrm{P} \mathrm{mg} / \mathrm{dL}$ & $2.7-5$ & $8.76 \pm 0.39^{\mathrm{a}}$ & $4.73 \pm 0.35^{\mathrm{b}}$ & $4.16 \pm 0.23^{\mathrm{bc}}$ & $3.86 \pm 0.18^{\mathrm{bc}}$ & $3.70 \pm 0.14^{\mathrm{c}}$ & $3.60 \pm 0.13^{\mathrm{c}}$ & $3.59 \pm 0.12^{\mathrm{c}}$ \\
\hline $\mathrm{TP} \mathrm{mg} / \mathrm{dL}$ & $6-7.5$ & $7.30 \pm 0.56$ & $7.02 \pm 0.37$ & $6.79 \pm 0.33$ & $6.70 \pm 0.34$ & $6.60 \pm 0.31$ & $6.72 \pm 0.29$ & $6.74 \pm 0.25$ \\
\hline ALB mg/dL & $2.5-4.2$ & $3.12 \pm 0.16$ & $3.24 \pm 0.10$ & $3.14 \pm 0.10$ & $3.04 \pm 0.10$ & $2.96 \pm 0.13$ & $3.02 \pm 0.11$ & $3.03 \pm 0.09$ \\
\hline $\mathrm{A} / \mathrm{G} \mathrm{mg} / \mathrm{dL}$ & $0.5-1.3$ & $0.76 \pm 0.08$ & $0.77 \pm 0.05$ & $0.84 \pm 0.05$ & $0.90 \pm 0.04$ & $0.90 \pm 0.05$ & $0.88 \pm 0.05$ & $0.86 \pm 0.05$ \\
\hline
\end{tabular}




\begin{tabular}{|c|c|c|c|c|c|c|c|c|}
\hline GLU mg/Dl & $50-100$ & $87.27 \pm 4.97$ & $91.66 \pm 2.95$ & $88.46 \pm 2.82$ & $86.40 \pm 2.53$ & $92.50 \pm 2.88$ & $84.90 \pm 2.36$ & $83.1 \pm 3.87$ \\
\hline ALT UI/L & $7-40$ & $63.01 \pm 6.95^{\mathrm{a}}$ & $53.63 \pm 3.38^{\mathrm{ab}}$ & $50.61 \pm 3.27^{\mathrm{b}}$ & $46.35 \pm 4.00^{\mathrm{b}}$ & $46.71 \pm 3.43^{\mathrm{b}}$ & $44.60 \pm 3.53^{\mathrm{b}}$ & $43.90 \pm 2.44^{\mathrm{b}}$ \\
\hline AST UI/L & $7-40$ & $48.76 \pm 5.09$ & $46.00 \pm 3.90$ & $45.30 \pm 4.06$ & $43.21 \pm 3.19$ & $42.64 \pm 2.52$ & $46.00 \pm 6.46$ & $40.80 \pm 2.49$ \\
\hline ALP UI/L & $5-110$ & $137.93 \pm 21.08^{\mathrm{a}}$ & $152.81 \pm 22.63^{\mathrm{ab}}$ & $156.39 \pm 21.69^{\mathrm{ab}}$ & $181.50 \pm 20.93^{\mathrm{b}}$ & $170.43 \pm 18.89^{\mathrm{ab}}$ & $178.40 \pm 18.30^{\mathrm{b}}$ & $188.20 \pm 17.68^{\mathrm{b}}$ \\
\hline BIL mg/dL & $0-0.7$ & $0.29 \pm 0.10$ & $0.26 \pm 0.08$ & $0.23 \pm 0.06$ & $0.21 \pm 0.05$ & $0.22 \pm 0.06$ & $0.21 \pm 0.05$ & $0.20 \pm 0.04$ \\
\hline CHOL mg/dL & $140-240$ & $318.58 \pm 24.39$ & $311.45 \pm 23.24$ & $332.56 \pm 24.82$ & $332.80 \pm 28.04$ & $335.70 \pm 25.57$ & $334.00 \pm 23.35$ & $332.30 \pm 24.19$ \\
\hline
\end{tabular}

2 Table 5. Biochemical parameters measured during the study: blood urea nitrogen (BUN), creatinine (CREA), phosphorus (P), total 3 protein (TP), albumin (ALB), albumin/globulin (A/G), glucose (GLU), alanine aminotransferase (ALT), aspartate aminotransferase 4 (AST), alkaline phosphatase (ALP), bilirubin (BIL), cholesterol (CHOL). Data are expressed as mean and standard error of the mean 5 (SEM); T: time in days. Means with different superscript letters $(\mathrm{a}, \mathrm{b}, \mathrm{c})$ indicate significant differences $(\mathrm{P}<0.05)$ among experimental 6 times within each group per each investigated parameter. 


\section{Table 6(on next page)}

Venous haemogas analysis parameters (serum bicarbonate $\left(\mathrm{HCO}_{3}\right)$ and ionized calcium (iCa)) measured during the study at regular intervals. Data are expressed as mean and standard error of the mean (SEM); T: time in days. In the T group, means with 


\begin{tabular}{|c|c|c|c|c|c|c|c|c|c|}
\hline Parameter & Laboratory standard reference range & T0 & T30 & T60 & T90 & T120 & T150 & T180 \\
\hline \multicolumn{7}{|c|}{ Parameters group C } \\
\hline HCO3- mmol/L & $18-24$ & $16.86 \pm 1.38$ & $16.93 \pm 1.30$ & $16.78 \pm 1.17$ & $16.56 \pm 1.02$ & $16.64 \pm 0.99$ & $16.58 \pm 0.89$ & $16.36 \pm 0.81$ \\
\hline iCa mmol/L & $1.29-1.41$ & $1.27 \pm 0.06$ & $1.27 \pm 0.06$ & $1.27 \pm 0.06$ & $1.27 \pm 0.06$ & $1.26 \pm 0.06$ & $1.25 \pm 0.05$ & $1.25 \pm 0.06$ \\
\hline \multicolumn{7}{|c|}{ Parameters group T } \\
\hline HCO3- mmol/L
\end{tabular}

2 Table 6. Venous haemogas analysis parameters (serum bicarbonate $\left(\mathrm{HCO}_{3}\right)$ and ionized calcium (iCa)) measured during the study at 3 regular intervals. Data are expressed as mean and standard error of the mean (SEM); T: time in days. In the T group, means with different 4 superscript letters $(a, b, c)$ indicate significant differences $(p<0.05)$ among the experimental times. 


\begin{tabular}{|c|c|c|}
\hline Patients & T0 & T180 \\
\hline 1 & 16,8 & 17,3 \\
\hline 2 & 17,0 & 17,5 \\
\hline 3 & 16,4 & 17,1 \\
\hline 4 & 16,4 & 17,7 \\
\hline 5 & 16,8 & 17,1 \\
\hline 6 & 15,9 & 17,0 \\
\hline 7 & 15,8 & 17,1 \\
\hline 8 & 15,8 & 16,7 \\
\hline 9 & 16,0 & 16,8 \\
\hline 10 & 15,6 & \\
\hline
\end{tabular}

14 Table 6B. Venous haemogas analysis parameters (serum bicarbonate, $\mathrm{HCO}_{3}$ ) measured at the beginning (T0) and the end (T180) of the study in the single patients from the $\mathrm{T}$ group. 
Table 7 (on next page)

Urinary parameters measured during the study. Urine protein-to-creatinine (UPC), urinary specific gravity(SG), urine protein (UP $\mathrm{mg} / \mathrm{dL}$ ). 


\begin{tabular}{|c|c|c|c|c|c|c|c|c|c|}
\hline Parameter & Laboratory standard reference range & T0 & T30 & T60 & T90 & T120 & T150 & T180 \\
\hline \multicolumn{7}{|c|}{ Parameters group C } \\
\hline UPC & $<0.5$ & $0.70 \pm 0.09$ & $0.73 \pm 0.04$ & $0.75 \pm 0.07$ & $0.84 \pm 0.06$ & $0.68 \pm 0.04$ & $0.74 \pm 0.06$ & $0.80 \pm 0.08$ \\
\hline UP mg/dL & $0-150$ & $223.57 \pm 23.33$ & $264.17 \pm 28.15$ & $272.14 \pm 31.75$ & $253.33 \pm 29.56$ & $239.38 \pm 24.55$ & $249.44 \pm 23.18$ & $266.88 \pm 25.23$ \\
\hline SG & $1020-1040$ & $1016.43 \pm 1.59$ & $1014.17 \pm 2.22$ & $1012.14 \pm 1.31$ & $1015.56 \pm 0.56$ & $1015.63 \pm 1.39$ & $1014.44 \pm 1.55$ & $1013.13 \pm 0.86$ \\
\hline & & & & & \\
\hline UPC & 0.5 & $0.63 \pm 0.06$ & $0.61 \pm 0.04$ & $0.65 \pm 0.04$ & $0.67 \pm 0.04$ & $0.67 \pm 0.03$ & $0.67 \pm 0.03$ & $0.65 \pm 0.03$ \\
\hline UP mg/dL & $0-150$ & $314.30 \pm 33.91$ & $290.10 \pm 25.71$ & $304.00 \pm 23.69$ & $302.40 \pm 24.02$ & $314.80 \pm 25.16$ & $302.50 \pm 22.93$ & $301.11 \pm 18.09$ \\
\hline SG & $1020-1040$ & $1014.80 \pm 1.74$ & $1016.50 \pm 1.30$ & $1014.50 \pm 1.39$ & $1015.50 \pm 1.39$ & $1016.00 \pm 1.00$ & $1018.00 \pm 1.11$ & $1017.50 \pm 1.54$ \\
\hline
\end{tabular}

2 Table 7. Urinary parameters measured during the study. Urine protein-to-creatinine (UPC), urinary specific gravity (SG), urine 3 protein (UP $\mathrm{mg} / \mathrm{dL})$. 
Figure 1

Serum phosphorus (P) concentration at days $0,30,60,90,120,150$ and 180 $(* * * p<0.001)$

\section{Serum P}

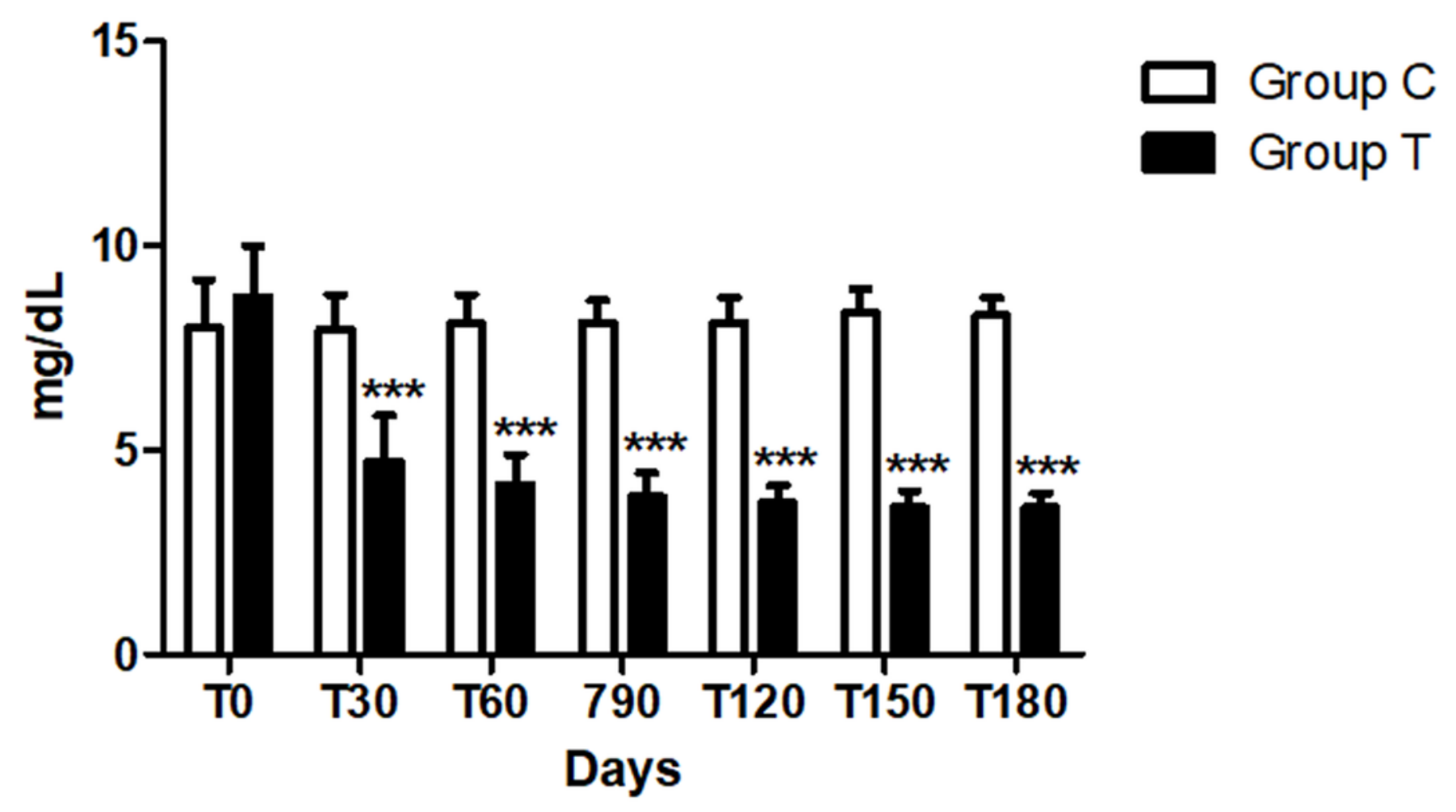


Figure 2

Serum ionized calcium (iCa) concentration at days $0,30,60,90,120,150$ and 180 $(* * p<0.01)$

\section{Serum iCa}

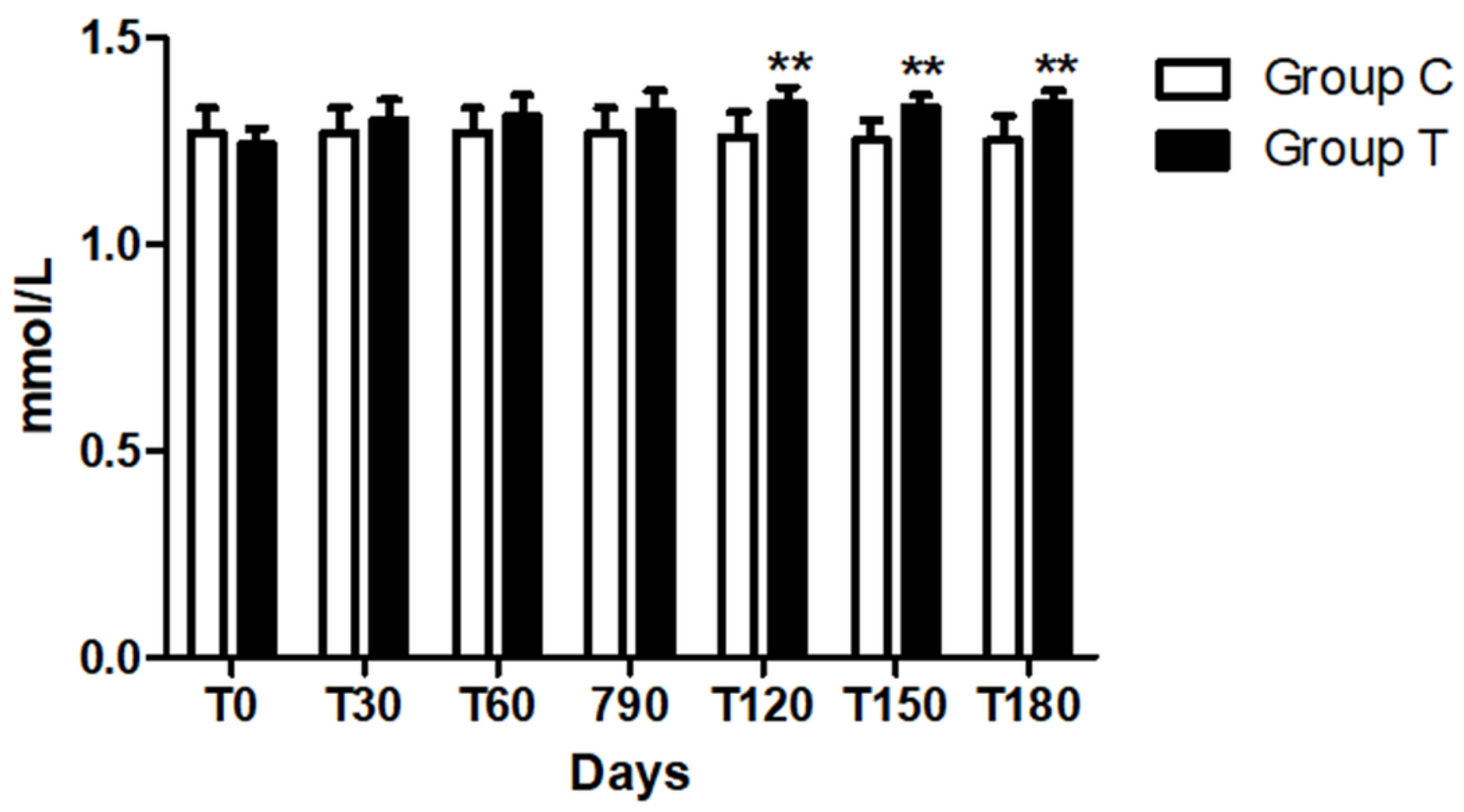


Figure 3

Serum bicarbonate $\left(\mathrm{HCO}_{3}\right)$ concentration at days $0,30,60,90,120,150$ and 180 $\left({ }^{*} p<0.05\right)$.

\section{Serum $\mathrm{HCO}_{3}$}

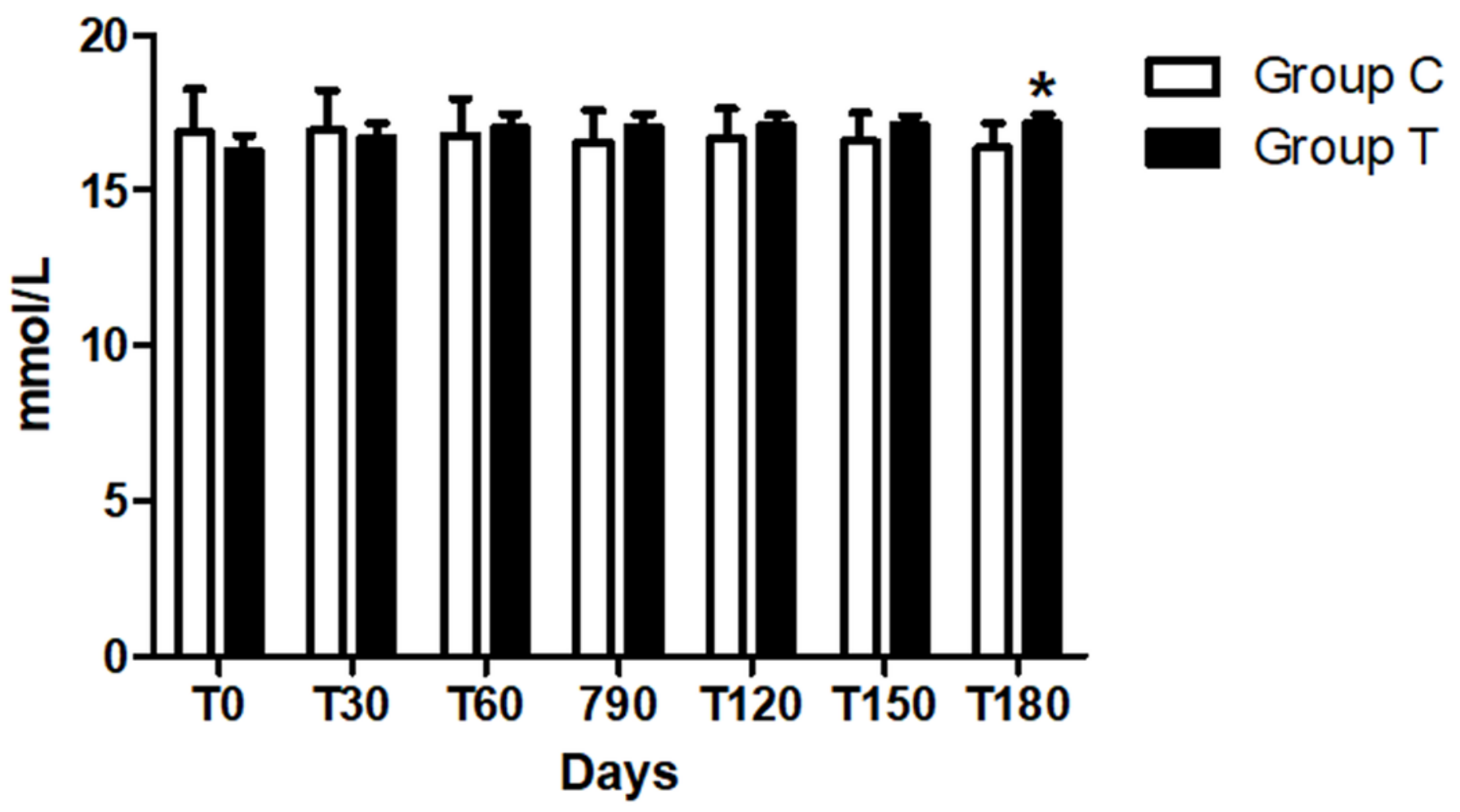


Figure 4

Urine protein/creatinine (UPC) at days $30,60,90,120,150$ and $180\left({ }^{*} p<0.05\right.$, $* * p<0.01$ )

\section{UPC}

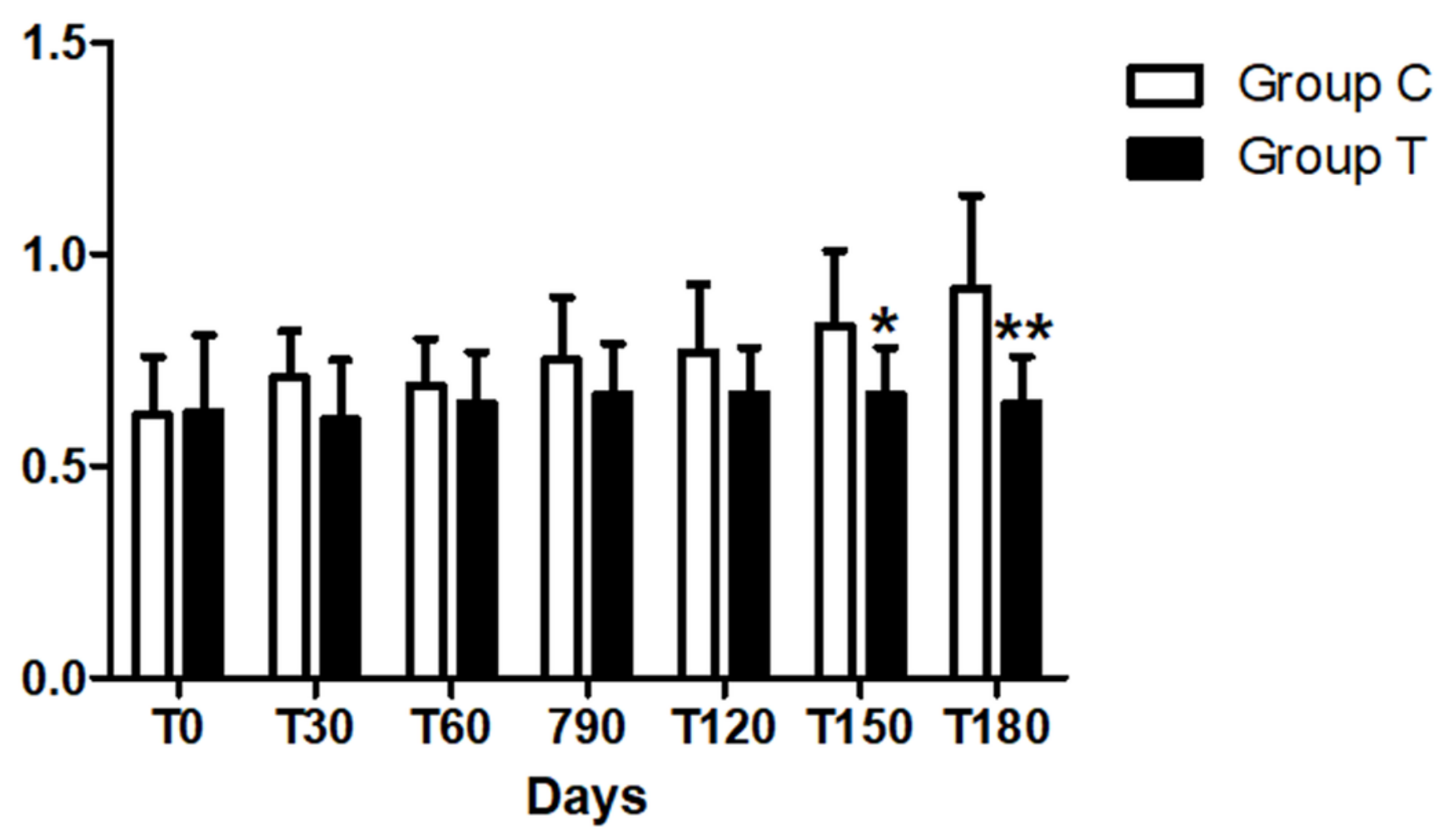

